\title{
Callus induction in pineapple (Ananas comosus L.) cv. Moris and Josapine.
}

\begin{abstract}
The induction of callus from Meristemic Globular Bodies (MGB) of two pineapple cultivars, namely Moris and Josapine, under six concentration levels of auxin NAA and six concentration levels of 2,4-D in Murashige and Skoog solid media, was investigated. 2,4-D auxin treatments failed to induce calli in both cultivars. However, 53.71, 75.19 and $85.93 \mu \mathrm{M}$ levels of auxin NAA caused calli induction in Moris while levels 32.22, 53.71 and $75.19 \mu \mathrm{M}$ also induced calli Josapine. The percentage of MGB calli formation increased with increasing time of culturing. At 6 weeks of culturing, $83 \%$ of Moris MGB explants formed calli on $85.93 \mu \mathrm{M}$ NAA, while $50 \%$ of Josapine MGB explants formed calli on $53.71 \mu \mathrm{M}$ NAA. Calli cultures have been an essential tool in the in vitro selection of desirable plants under manipulated conditions and from in vitro mutations via somaclonal variation. More importantly, calli are increasingly used for the application of cellular level genetic modification techniques such as the Agrobacterium-mediated transformation, particle bombardment and protoplast isolation and fusion. In this study, auxin NAA successfully initiated and proliferated calli in Moris and Josapine globular meristemic cultures.
\end{abstract}

Keyword: Callus induction; Pineapple; NAA; 2,4-D. 